\title{
Promoting social plasticity in developmental disorders with non-invasive brain stimulation techniques
}

\author{
Paulo S. Boggio *, Manish K. Asthana, Thiago L. Costa, Cláudia A. Valasek and \\ Ana A. C. Osório \\ Social and Cognitive Neuroscience Laboratory and Developmental Disorders Program, Center for Health and Biological \\ Sciences, Mackenzie Presbyterian University, Sao Paulo, Brazil
}

\section{OPEN ACCESS}

Edited by:

Pablo Billeke,

Universidad del Desarrollo, Chile

Reviewed by:

Zhihui Yang,

University of Florida, USA

Satyaprakash Nayak,

Pfizer Inc., USA

*Correspondence:

Paulo S. Boggio,

Social and Cognitive Neuroscience

Laboratory and Developmental

Disorders Program, Center for Health and Biological Sciences, Mackenzie

Presbyterian University, Rua Piaui,

$181,10^{\circ}$ andar, Sao Paulo,

SP 01241-001, Brazil psboggio@gmail.com

Specialty section:

This article was submitted to Systems Biology, a section of the journal

Frontiers in Neuroscience

Received: 05 February 2015

Accepted: 05 August 2015

Published: 01 September 2015

Citation:

Boggio PS, Asthana MK, Costa TL, Valasek CA and Osório AAC (2015)

Promoting social plasticity in developmental disorders with non-invasive brain stimulation techniques. Front. Neurosci. 9:294. doi: 10.3389/fnins.2015.00294
Being socially connected directly impacts our basic needs and survival. People with deficits in social cognition might exhibit abnormal behaviors and face many challenges in our highly social-dependent world. These challenges and limitations are associated with a substantial economical and subjective impact. As many conditions where social cognition is affected are highly prevalent, more treatments have to be developed. Based on recent research, we review studies where non-invasive neuromodulatory techniques have been used to promote Social Plasticity in developmental disorders. We focused on three populations where non-invasive brain stimulation seems to be a promising approach in inducing social plasticity: Schizophrenia, Autism Spectrum Disorder (ASD) and Williams Syndrome (WS). There are still very few studies directly evaluating the effects of transcranial direct current stimulation (tDCS) and transcranial magnetic stimulation (TMS) in the social cognition of these populations. However, when considering the promising preliminary evidences presented in this review and the limited amount of clinical interventions available for treating social cognition deficits in these populations today, it is clear that the social neuroscientist arsenal may profit from non-invasive brain stimulation techniques for rehabilitation and promotion of social plasticity.

Keywords: brain stimulation, neuromodulation, developmental disorders, autism, Williams syndrome, schizophrenia, social cognition

\section{Introduction}

One of the crucial aspects of human beings is the urge to be socially connected. From the need to be cared for (newborn babies) to the pleasure of being in a romantic relationship, being socially connected directly impacts our basic needs and survival. People with deficits in social cognition might present abnormal behaviors in our highly social-dependent world. Along this line, a lot of effort has been allocated to studies on neuropsychiatric disorders. More specifically, several studies have attempted to understand the role of social cognition in developmental disorders by examining their behavioral manifestations. Also, electrophysiological and neuroimaging studies have taught us about the functional and structural neuronal basis of different developmental disorders.

We have also been learning about brain plasticity-from the good, bad, and ugly. The negative impact of lesions and the positive impact of enriched environments on cognitive functions are 
few examples of it. Based on recent research, we propose a review of the use of non-invasive neuromodulatory techniques to promote Social Plasticity (i.e., the modulation of the neural substrate associated with social cognition aiming for more adaptive social interactions) in some developmental disorders. Particularly, we will focus on Transcranial Magnetic Stimulation (TMS) and Transcranial Direct Current Stimulation (tDCS).

The impact of social cognition alterations or deficits faced by some clinical populations is well known. Nonetheless, most research on non-invasive brain stimulation techniques in the treatment and rehabilitation of clinical populations has focused on other aspects of cognition (such as executive functions, attention, or memory). In this scenario, such techniques might also play a crucial role in addressing impairments in social cognition and behavior, as we will argue below. As social neuroscience is a relatively new field and the use of non-invasive brain stimulation techniques in this context is even more recent, it should come as no surprise that only very few works have used these techniques to approach these issues. In this paper we will emphasize three populations where non-invasive brain stimulation seems to be a promising technique in inducing social plasticity: Schizophrenia, Autism Spectrum Disorder (ASD) and Williams Syndrome (WS). Our main goal is to emphasize how non-invasive brain modulation may be a valuable strategy in this context and how the plasticity of social cognition and behavior in clinical populations is a possible and relevant goal for contemporary research.

\section{Autism Spectrum Disorder}

There are certainly more investigations of social cognition in ASD than in schizophrenia and WS and it seems fair to say (although a systematic comparison is lacking) that there are more numerous and more effective interventions for social cognition in ASD than in the other two disorders. A review of therapies available for children with ASD can be found in Warren et al. (2011). Among the recent and still experimental ones we highlight intranasal oxytocin and the Early Start Denver Model (all reviewed in Canitano, 2014). Intranasal oxytocin has been shown to increase patients' gaze fixation on the most socially informative regions of the face and to increase interactions with partners and feelings of trust in an experimental paradigm (Andari et al., 2010). In fact, a systematic review of seven studies evaluating oxytocin interventions in ASD found significant effects in all studies but one (Preti et al., 2014). A behavioral technique that has also showed promising results is the Early Start Denver Model, an applied behavior analysis based educational intervention focused on building communicative abilities, enhancing social attention and reinforcing social interaction (e.g., Fulton et al., 2014).

These interventions and results show that it is indeed possible to induce plasticity of social functioning in ASD patients. Nonetheless, ASD expression is very heterogeneous, comprising a whole spectrum of different symptoms in different severities. The outcomes of possible interventions tend to be very variable too (e.g., Warren et al., 2011; Magiati et al., 2014; Preti et al.,
2014). Also, side effects of medical interventions might be significant (e.g., Warren et al., 2011). In this context new interventions must be developed, including combinations of complementary strategies. Until now, few studies involving neuromodulation techniques in patients with autism have been conducted and most part used Transcranial Magnetic Stimulation (TMS) (see Table 1).

Some studies with TMS focus on motor functions, taking into account assumptions about partial impairment of the mirror neuron system (MNS). The MNS is responsible for understanding intention and imitation of behavior observed in other subjects. In addition to being involved in language development (Williams et al., 2001).

Studies with observation and/or mentoring of motor actions have been used to study the functions of the MNS. From the studies conducted in healthy volunteers (Fadiga et al., 1999; Fourkas et al., 2006; Cesari et al., 2011), it can be seen that TMS provides accurate measurements of cortical excitability and confirms the increased activity as well as motor threshold reduction in motor cortical areas during observation and mental imagery related to motor function. Considering these data, some authors have investigated the relationship between motor imagery and cortical excitability in ASD. Théoret et al. (2005) showed that cortical excitability in autism is significantly lower compared to a control group during a finger movement observation task. The observation of the movements of the fingers facilitated motor evoked potential generated by TMS in the control group but not in the autism group. Other studies with single-pulse TMS show that the observation of specific motor movements or observations of painful movements do not induce corticospinal modulation in patients with ASD (MinioPaluello et al., 2009; Enticott et al., 2012). Similar results were observed by Puzzo et al. (2009). After measurement of motor evoked potentials MEPs during observation of videos with hand or mouth movements, static images of hand or mouth, and white screen images in patients with high and low traits of autism, no difference between groups was observed for the white screen condition. However, participants with low traits of autism showed higher MEP amplitudes during observation of actions videos compared to static images. This result was not observed in patients with high traits of autism, which exhibited similar MEP amplitude in the observation of actions and observation of static images (Puzzo et al., 2009).

Another possibility would be the use of TMS application as a social cognitive rehabilitation tool. In a preliminary study, Sokhadze et al. (2009) rTMS applied low frequency $(0.5 \mathrm{~Hz})$ in the left DLPFC in 8 patients with autism, 2 times per week for 3 weeks. The results showed changes in relation to electrophysiological measures such as the reduction in the amplitude and latency of the P3 component to non-target stimuli. In addition to the normalization of $\mathrm{P} 3$ component, it was also observed decreased gamma frequency in frontal and parietal regions to non-target stimuli. Regarding clinical outcomes, decreased ritualistic behavior was observed as measured by scales and caregivers reported a decrease in repetitive behavior and obsessive-compulsive behaviors. Subsequent studies have replicated these results, showing the reduction of repetitive 


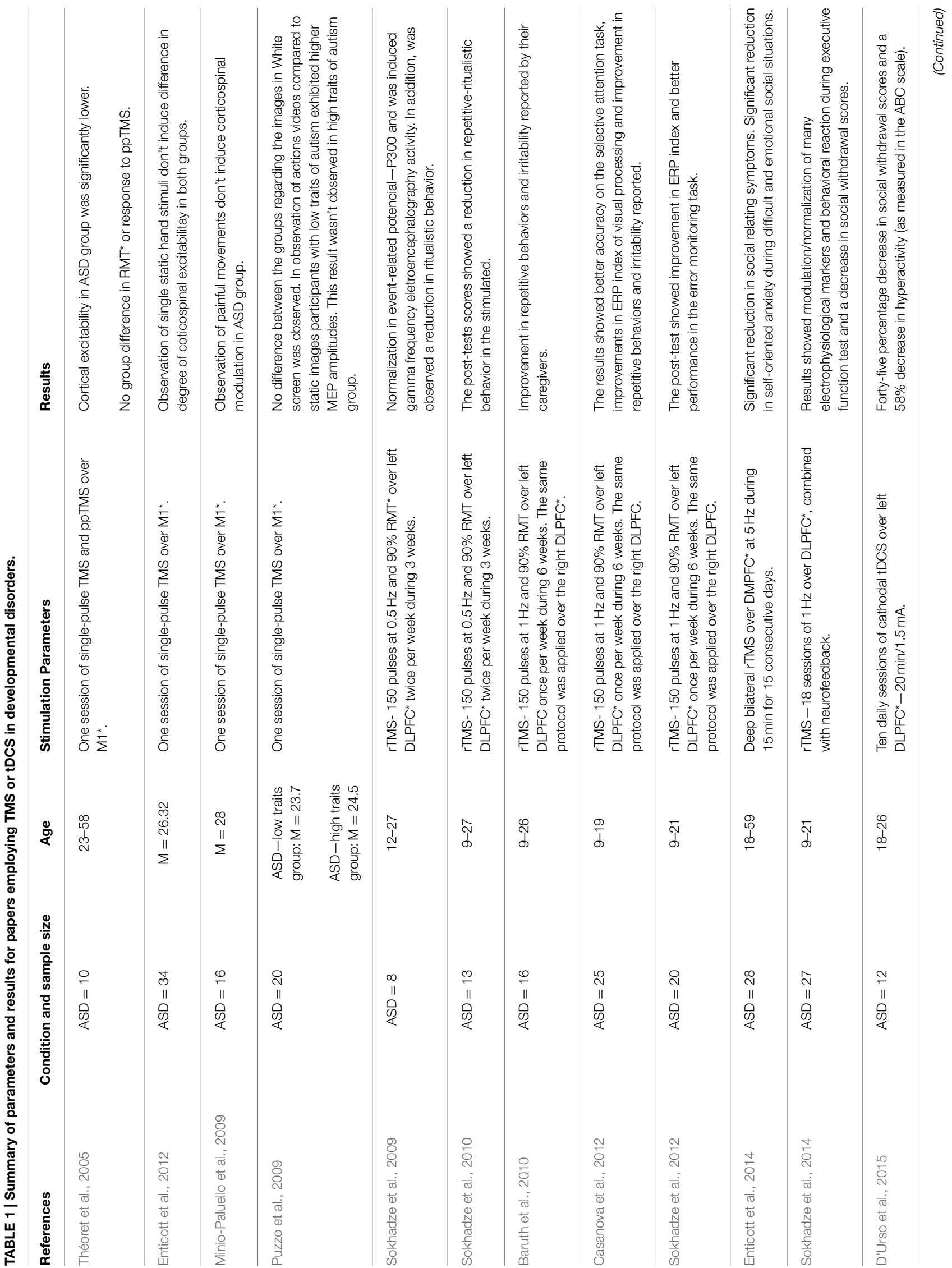


behaviors and irritability (Baruth et al., 2010; Sokhadze et al., 2010). Still on the observation of improvement in these behaviors, Casanova et al. (2012) used a protocol of rTMS $(1 \mathrm{~Hz})$ on CLPFC for 12, 6 weeks over left hemisphere and 6 weeks over right hemisphere. The results showed better performance on the selective attention task and improve behaviors of irritability and repetition. By using the same protocol, Sokhadze et al. (2012) also found improved performance in the error-monitoring task. In consonance with that, Sokhadze et al. (2014) have observed modulation of many electrophysiological markers and a decrease in social withdrawal scores after 18 sessions of rTMS combined with neurofeedback in ASD patients. Finally, Enticott et al. (2014) showed improvement of social skills and anxiety symptoms in 28 patients with autism (high functioning and Asperger's), after application of deep bilateral rTMS in PFC medial dorsal of $5 \mathrm{~Hz}$ for $15 \mathrm{~min}$ for 15 consecutive days. This improvement was maintained after 1 month of stimulation.

These few studies showing substantial TMS effects in social cognition in ASD might be directly related to the well-known inhibitory/excitatory neural dynamics that seems to be affected in ASD and this phenomenon seems to take place at least in part through dysfunctional glutamate and GABA neurotransmitter systems (Won et al., 2013). In fact, reduced GABA synthesis and dysfunctional GABA receptors were found in autistic brains (Fatemi et al., 2010). Abnormally low levels of glutamine and glutamate were also found in autistic children (Rolf et al., 1993). Given the fact that tDCS is known to influence glutamate and GABA concentrations depending on stimulation polarity (e.g., Filmer et al., 2014), it is reasonable to expect that this tool might be helpful in ASD rehabilitation.

Given the aforementioned advantages of tDCS relative to TMS, patients would face fewer restrictions when undergoing a treatment with the former technique, when compared to the latter. Therefore, more investigations with tDCS in ASD must be done, in order to evaluate if similar effects to the ones induced by rTMS could be achieved. Here we highlight one example of substantial tDCS modulation of structures that were also successfully modulated by TMS in previous research, and that are relevant to the treatment of ASD. Theory of mind was found to be modulated by TMS of the right temporal parietal junction (Young et al., 2010). Later, Santiesteban et al. (2012) demonstrated improvements in theory of mind and increased social ability after tDCS of the temporal parietal junction. Both experiments were performed with participants with typical development.

Childhood is arguably the most critical windows for interventions and therapy in ASD (e.g., Warren et al., 2011). The fact that the use of tDCS in children is still controversial and poorly understood (with almost no studies on safety and tolerability, e.g., Gillick et al., 2015) may be considered a drawback for its application in ASD. Nonetheless, tDCS can be applied to young adults and this is still a reasonable moment for interventions, especially if one considers that ASD symptoms will frequently still be present throughout adulthood (e.g., Tobin et al., 2014).

Lastly, a recent case study with an adult diagnosed with ASD since the age of 2 years supports all that has been proposed 
here. D'Urso et al. (2015) report what seems to be the first investigation to use tDCS in an ASD patient. These authors have delivered 10 daily sessions of cathodal tDCS to the left DLPFC in a patient that was non-respondent to pharmacological treatments and showed limited outcomes for behavioral interventions. The patient showed a $45 \%$ decrease in social withdrawal scores and a $58 \%$ decrease in hyperactivity (as measured in the ABC scale). The results by D'Urso et al. (2015) are very encouraging and support the need for more tDCS based investigations in all aspects of ASD, not only social cognition.

\section{Schizophrenia}

In conditions such as schizophrenia, deficits in social cognition are known to take place and to be determinants of patients' limits in functional abilities. These deficits include impairments in Theory of Mind strategies, emotional content processing, among others (Pinkham et al., 2003). In fact, in a recent meta-analysis that investigates the relations between neurocognition (defined in this context as attention, memory, executive functions, etc.,) and social cognition in schizophrenia, Fett et al. (2011) have found that social cognition deficits were stronger determinants of patients' functional outcomes than neurocognition deficits. Another recent work has shown that these social cognition deficits are also present during remission and are only weakly correlated to neurocognition deficits (Mehta et al., 2013). These findings draw attention to the crucial role played by social cognition deficits in schizophrenia and the need to focus on social cognition in the research and treatment of these patients.

As some authors have proposed (reviewed in a meta-analysis by McGurk et al., 2007), cognitive interventions in schizophrenia are generally only able to improve functionality to a small or medium extent. As there is evidence that these social cognition impairments are present even during remission, more effort has to be put into improving this crucial aspect in this population. Therefore, non-invasive brain stimulation interventions may be very valuable and as we will present ahead, there is compelling evidence (although with only few investigations yet) that this is indeed a promising strategy.

Three meta-analyses-one on the effects of TMS in diminishing auditory hallucinations and two on the TMS use for treating negative symptoms-support that TMS is a valuable intervention for treating schizophrenia symptoms (Aleman et al., 2007; Freitas et al., 2009; Shi et al., 2014). Aleman et al. (2007) have found substantial evidence for the applicability of TMS for the treatment of auditory hallucinations in schizophrenia but no significant effect on a general index of psychotic symptoms after reviewing 10 studies. Nonetheless, Freitas et al. (2009) and Shi et al. (2014) have found significant effects of TMS on decreasing negative symptoms, a finding that supports the adequacy of this technique in treating social symptoms in schizophrenia. The rationale in most of the studies is that there is a deficit of intracortical inhibition in schizophrenia and that inhibitory non-invasive brain stimulation might improve this aspect of the disease (see Hasan et al., 2013 for a review of intracortical inhibition in schizophrenia). In fact, there is substantial evidence for a reduced density of GABA interneurons in schizophrenia and some evidence that this intracortical inhibition deficit in schizophrenia (as assessed by TMS) is significantly correlated to deficits in social cognition (Mehta et al., 2014).

tDCS has also been used in few investigation with schizophrenic patients and is showing promising outcomes. A recent systematic review by Brunoni et al. (2014) has found a number of investigations where tDCS was shown to improve auditory or visual hallucinations in schizophrenia. Two of these works have also reported a global improvement in symptoms experienced by the patients (Brunelin et al., 2012 and a case report by Palm et al., 2013). The study of Brunelin et al. (2012), for example, observed a significant reduction of auditory hallucinations (average reduction of $31 \%$ ), negative and positive symptoms that was visible for up to 3 months after the tDCS sessions. Palm et al. (2013) has also found a decrease in negative symptoms and auditory hallucinations after 2 weeks of tDCS. If an improvement in negative symptoms is observed, some improvement in sociability is also expected.

The improvements reported in these studies are in accordance with what was observed in the computational models of current flow for the tDCS montages most frequently employed in this field. Brunoni et al. (2014) identified that the electrode montage used most frequently in these investigations targets the left dorsolateral prefrontal cortex (an area associated with negative symptoms in schizophrenia) and that the current generally flows through deeper structures involved in schizophrenic symptoms (as the cingulated cortex, insula, basal ganglia, and hippocampus).

All the evidence mentioned here supports the need and relevance of more studies on brain stimulation and social cognition in schizophrenia. As we have argued above, social cognition is impaired in schizophrenia and it seems to be a stronger determinant of patients' functional outcomes than neurocognition (Fett et al., 2011). Therefore, it is surprising and noteworthy how there are still very few investigations of the non-invasive brain stimulation effects in social functioning in schizophrenic These techniques seem to have an important role as tools to modulate positive and negative symptoms in schizophrenia. This is particularly relevant when considering that many participants are drug resistant or present medication refractory schizophrenia. In these cases, nonpharmacological interventions as TMS and tDCS might be great alternatives.

\section{Williams Syndrome}

Williams syndrome (WS) is a rare neurodevelopmental disorder caused by a multiple gene deletion on chromosome $7 q 11.23$ (Korenberg et al., 2000). Apart from a set of medical, physical, and cognitive features (including mild to moderate intellectual disability), individuals with the disorder are commonly described as having the opposite social profile of individuals with ASD (Karmiloff-Smith et al., 2012). Indeed, several studies have reported exacerbated interest in social stimuli and excessive social approach (including to strangers) as remarkable features present from early infancy into adulthood-a tendency termed hypersociability (Jones et al., 2000; Mervis et al., 2001; Laing et al., 2002; Riby and Hancock, 2008, 2009). However, these seemingly endearing personality features often pose important threats to 
these individuals, putting them at risk in unsupervised social interactions. For instance, studies showed that persons with WS tend to rate unfamiliar faces as significantly more approachable than age-matched controls (Jones et al., 2000; Martens et al., 2009). Furthermore, a recent study found that these individuals are more likely to consider approaching and ultimately choose to approach untrustworthy faces significantly more than controls matched in chronological age (Martens et al., 2012). These same behavioral tendencies studied in laboratory settings are reported to occur in everyday interactions, with a significant negative impact for individuals with this syndrome. In particular, they experience greater rates of sexual abuse when compared to samples with intellectual disability (Rosner et al., 2004). In a recent paper by Fisher et al. (2013) parents of individuals with WS reported higher levels of social vulnerability-particularly lower risk awareness by their children-than parents of individuals with ASD or Down syndrome. Consistent with these reports, some authors highlight how hypersociability goes alongside difficulties in regulating social behaviors (Jawaid et al., 2012; Riby et al., 2014), drawing attention to the deleterious effects of hypersociability for individuals with WS and their families.

Several hypotheses have been advanced to explain this trait, namely the amygdala hypothesis and the prefrontal hypothesis (Jawaid et al., 2008; Capitão et al., 2011). The former suggests that structural or functional alterations in the amygdala might account for the difficulties in recognizing threat-particularly in social interactions-, while the latter proposes that despite being able to detect social threat, individuals with WS are unable to inhibit approach behaviors due to prefrontal impairments. The results reported so far seem to suggest that these hypotheses might be complementary, rather than mutually exclusive (Mimura et al., 2010; Capitão et al., 2011). In this line, MeyerLindenberg et al. (2005) demonstrated the joint activation of the amygdala along with dorsolateral prefrontal cortex (DLPFC), medial prefrontal cortex (MPFC), and orbitofrontal cortex (OFC) in a threatening face matching task in healthy controls. In contrast, this pattern of joint activation was absent in a select sample of WS with intact cognitive abilities. More specifically, OFC was not selectively recruited and path analysis further showed that this region was not functionally connected to the amygdala-a result that is consonant with the strong social disinhibition seen in WS. In addition, abnormal interactions were found between MPFC and DLPFC in WS, which were consistent with a possible compensatory mechanism for the disrupted OFCamygdala circuit (Meyer-Lindenberg et al., 2005). In a subsequent study with the same sample, Munoz et al. (2010) reported further evidence of abnormal circuitry involved in the processing of social vs. non-social threat in WS, reinforcing these regions as likely involved in the emergence of hypersociability.

Together, these findings open new avenues for the use of neuromodulation, as the aforementioned brain regions afford possible target areas aimed at improving social cognition and reducing hypersociable tendencies in WS. So far, only sparse behavioral interventions (mostly case studies) have been implemented with these goals in mind, with inconsistent success (Klein-Tasman and Albano, 2007; Phillips and Klein-Tasman, 2009; Jawaid et al., 2012). Importantly, no studies have employed either TMS or tDCS in this clinical population. We argue that the successful use of these non-invasive and generally safe techniques in several other neurodevelopmental disorders (particularly those cited in this paper) provides encouragement for future studies to test its therapeutic potential in WS.

\section{Noteworthy Issues in Brain Stimulation}

The current review focuses on two of the most popular brain stimulation techniques: tDCS and TMS. These techniques differ in dynamics and mechanisms of action to a great extent. tDCS is associated with moderate modulation of the voltage gradient between the intracellular and extracellular medium, while TMS can induce action potentials. These techniques also differ in the focality of the stimulation and its potential outcomes. For recent reviews of parameters and mechanisms of action we refer to Stagg and Nitsche (2011) and Filmer et al. (2014) for tDCS and Pascual-Leone et al. (2000) and Miniussi and Rossini (2011) for TMS.

For the readers that are not familiar with non-invasive brain stimulation, we must highlight the safety of these techniques when standard parameters are used. For tDCS, extensive data suggest that this is a safe procedure that involves mild and transient adverse effects (Nitsche et al., 2008; Brunoni et al., 2012). In a systematic review of tDCS studies, Brunoni et al. (2012) found that $56 \%$ of the investigations mentioned adverse effects but these were limited to itching or tingling under the electrodes, headache, and discomfort. These adverse effects were also present in participants receiving sham tDCS, thus suggesting that the major cause of side effects may not be the current itself. Recent studies showed that tDCS does not induce elevations of a neuronal damage marker (neuron-specific enolase) and no edemas in humans and no maladaptive functional or structural changes were observed in evaluations using EEG and fMRI (Nitsche et al., 2004, 2008; Iyer et al., 2005). It is still not clear if repeated tDCS sessions during weeks and months can lead to undesirable side effects and more research on that matter is advised.

Although in some cases TMS might be associated with more severe adverse effects than tDCS, generally its adverse effects are mild and transient and the technique is considered safe when standard parameters are employed (e.g., Rossi et al., 2009). In general terms TMS protocols may be divided between the ones that use isolated single pulses of stimulation, the ones that use paired pulses separated by very brief intervals and the ones that use repetitive pulses at one specific temporal frequency that might range from a few seconds to minutes. Amongst these, repetitive TMS protocols are the ones with more long lasting effects and more significant therapeutic potential. It is also the stimulation modality that is associated with more significant adverse effects, and this is why many guidelines suggest it should be delivered in hospital facilities (Lefaucheur et al., 2014).

The ease of use, portability and minimal adverse effects associated with tDCS might be considered one of its advantages over TMS. In fact, in the field of pain treatment research, a systematic review by Zaghi et al. (2009) concluded that when compared to other neuromodulatory techniques (such as repetitive TMS or EDCS), tDCS is the most cost-effective treatment for central pain (considering treatments shorter than 
5 years). Nonetheless, each technique has its strengths and possible drawbacks. This must be considered carefully when planning each investigation. The functional and spatial focality of the two techniques is frequently a matter of debate, and in many cases there is no easy answer for which technique to employ. In fact, more research comparing these techniques is also advised.

Lastly, as the clinical conditions reviewed here are more frequently diagnosed first in children and adolescents, a brief mention to possible peculiar effects of non-invasive brain stimulation in the developing brain must be made. Until recently there were only few works on the effects of tDCS and TMS in children and adolescents. This was probably determined at least in part by ethical concerns, given the fact that the developing brain could be affected in nonintuitive ways by these interventions. Recent advances in our understanding of mechanisms of action and outcomes of non-invasive brain stimulation techniques, paired with a few successful investigations in children are changing this scenario.

A recent review of TMS studies in children has suggested that this intervention is well tolerated and promising (Frye et al., 2008). Nonetheless the authors have suggested that specific guidelines need to be created for children. The same is true for tDCS. Some recent works have suggested that tDCS might be effective and well tolerated in children (Minhas et al., 2012; Andrade et al., 2014), but more investigations of this subject are still lacking. It is also important to note that in many cases, a rationale that was proven to be successful for stimulation in adults might not turn out to have the same outcomes in the developing brain and more research in this population is still needed.

\section{Conclusions and Future Directions}

This article presents evidence that neuromodulation techniques may become important interventional tools in social deficits. Research focused on the understanding of the mechanisms of

\section{References}

Aleman, A., Sommer, I. E., and Kahn, R. S. (2007). Efficacy of slow repetitive transcranial magnetic stimulation in the treatment of resistant auditory hallucinations in schizophrenia: a meta-analysis. J. Clin. Psychiatry 68, 416-421. doi: 10.4088/JCP.v68n0310

Andari, E., Duhamel, J. R., Zalla, T., Herbrecht, E., Leboyer, M., and Sirigu, A. (2010). Promoting social behavior with oxytocin in high-functioning autism spectrum disorders. Proc. Natl. Acad. Sci. U.S.A. 107, 4389-4394. doi: 10.1073/pnas.0910249107

Andrade, A. C., Magnavita, G. M., Allegro, J. V. B. N., Neto, C. E. B. P., Lucena, R. D. C. S., and Fregni, F. (2014). Feasibility of transcranial direct current stimulation use in children aged 5 to 12 years. J. Child Neurol. 29, 1360-1365. doi: 10.1177/0883073813503710

Brunoni, A. R., Amadera, J., Berbel, B., Volz, M. S., Rizzerio, B. G., and Fregni, F. (2012). A systematic review on reporting and assessment of adverse effects associated with transcranial direct current stimulation. Int. J. Neuropsychopharmachol. 14, 1133-1145. doi: 10.1017/S146114571 0001690 action of these techniques as well as on ways to direct their effects to enhance social cognition deficits will open new avenues in what we are calling Social Plasticity. As shown, there are few studies directly evaluating the effects of tDCS and TMS in populations such as autism, schizophrenia and WS. As regards to the social cognition deficits, these techniques are still in their infancy. With that, a research agenda in this area is indispensable. As central aspects, it is necessary to standardize procedures like the use of common assessment tools. It is also necessary to initially investigate the short-term effects of these techniques (for example, single session stimulation) and then move forward with investigations on the lasting effects of these tools. This care is critical considering the potential that these techniques have in the promotion of brain plasticity. Another key aspect relates to the large heterogeneity of the manifested symptoms. Taking autism as an example, the great diversity of manifestations within the spectrum makes you take precautions in the translation of knowledge from one study to another. Based on the studies of cognitive neuroscience, it is clear that noninvasive neuromodulation techniques are already part of the toolkit box of both research and rehabilitation domains. With the first evidences presented in this review, it is flagrant the need to insert these techniques in the social neuroscientist arsenal particularly focused on rehabilitation and promotion of social plasticity.

\section{Author Contributions}

All authors contributed to manuscript writing and approved the final version of the manuscript for submission.

\section{Acknowledgments}

MA is supported by a Post-doc grant (PNPD/CAPES-2014). $\mathrm{CV}$ is supported by a $\mathrm{PhD}$ grant (FAPESP 13/26500-0). TC is a FAPESP postdoctoral fellow (FAPESP 2014/11668-5). PB is a $\mathrm{CNPq}$ research fellow. This research was supported by MackPesquisa.
Baruth, J. M., Casanova, M. F., El-Baz, A., Horrell, T., Mathai, G., Sears, L., et al. (2010). Low-frequency repetitive transcranial magnetic stimulation (rTMS) modulates evoked-gamma frequency oscillations in autism spectrum disorder (ASD). J. Neurother. 14, 179-194. doi: 10.1080/10874208.2010.501500

Brunelin, J., Mondino, M., Haesebaert, F., Saoud, M., Suaud-Chagny, M. F., and Poulet, E. (2012). Efficacy and safety of bifocal tDCS as an interventional treatment for refractory schizophrenia. Brain Stimul. 5, 431-432. doi: 10.1016/j.brs.2011.03.010

Brunoni, A. R., Shiozawa, P., Truong, D., Javitt, D. C., Elkis, H., Fregni, F., et al. (2014). Understanding tDCS effects in schizophrenia: a systematic review of clinical data and an integrated computation modeling analysis. Expert Rev. Med. Devices 11, 383-394. doi: 10.1586/17434440.2014.911082

Canitano, R. (2014). New experimental treatments for core social domain in autism spectrum disorders. Front. Pediatr. 2:61. doi: 10.3389/fped.2014. 00061

Capitão, L., Sampaio, A., Férnandez, M., Sousa, N., Pinheiro, A., and Gonçalves, Ó. F. (2011). Williams syndrome hypersociability: a neuropsychological study of the amygdala and prefrontal cortex hypotheses. Res. Dev. Disabil. 32, 1169-1179. doi: 10.1016/j.ridd.2011.01.006 
Casanova, M. F., Baruth, J. M., El-Baz, A., Tasman, A., Sears, L., and Sokhadze, E. (2012). Repetitive transcranial magnetic stimulation (rTMS) modulates eventrelated potential (ERP) indices of attention in autism. Transl. Neurosci. 3, 170-180. doi: 10.2478/s13380-012-0022-0

Cesari, P., Pizzolato, F., and Fiorio, M. (2011). Grip-dependent cortico-spinal excitability during grasping imagination and execution. Neuropsychologia 49, 2121-2130. doi: 10.1016/j.neuropsychologia.2011.04.019

D’Urso, G., Bruzzese, D., Ferrucci, R., Priori, A., Pascotto, A., Galderisi, S., et al. (2015). Transcranial direct current stimulation for hyperactivity and noncompliance in autistic disorder. World J. Biol. Psychiatry 16, 361-366. doi: 10.3109/15622975.2015.1014411

Enticott, P. G., Fitzgibbon, B. M., Kennedy, H. A., Arnold, S. L., Elliot, D., Peachey, A., et al. (2014). A double-blind, randomized trial of deep repetitive transcranial magnetic stimulation (rTMS) for Autism Spectrum Disorder. Brain Stimul. 7, 206-211. doi: 10.1016/j.brs.2013.10.004

Enticott, P. G., Kennedy, H. A., Rinehart, N. J., Tonge, B. J., Bradshaw, J. L., Taffe, J. R., et al. (2012). Mirror neuronactivity associated with social impairments but not age in autismo spectrum disorder. Biol. Psychiatry 71, 427-433. doi: 10.1016/j.biopsych.2011.09.001

Fadiga, L., Buccino, G., Craighero, L., Fogassi, L., Gallese, V., and Pavesi, G. (1999). Corticospinal excitability is specifcally modulated by motor imagery: a magnetic stimulation study. Neuropsychologia 37, 147-158. doi: 10.1016/S00283932(98)00089-X

Fatemi, S. H., Reutiman, T. J., Folsom, T. D., Rooney, R. J., Patel, D. H., and Thuras, P. D. (2010). mRNA and protein levels for GABAA $\alpha 4, \alpha 5, \beta 1$ and GABABR1 receptors are altered in brains from subjects with autism. J. Autism Dev. Disord. 40, 743-750. doi: 10.1007/s10803-009-0924-Z

Fett, A. K. J., Viechtbauer, W., Penn, D. L., van Os, J., and Krabbendam, L. (2011). The relationship between neurocognition and social cognition with functional outcomes in schizophrenia: a meta-analysis. Neurosci. Biobehav. Rev. 35, 573-588. doi: 10.1016/j.neubiorev.2010.07.001

Filmer, H. L., Dux, P. E., and Mattingley, J. B. (2014). Applications of transcranial direct current stimulation for understanding brain function. Trends Neurosci. 37, 742-753. doi: 10.1016/j.tins.2014.08.003

Fisher, M. H., Moskowitz, A. L., and Hodapp, R. M. (2013). Differences in social vulnerability among individuals with autism spectrum disorder, Williams syndrome, and Down syndrome. Res. Autism Spectr. Disord. 7, 931-937. doi: 10.1016/j.rasd.2013.04.009

Fourkas, A. D., Avenanti, A., Urgesi, C., and Aglioti, S. M. (2006). Corticospinal facilitation during first and third person imagery. Exp. Brain Res. 168, 143-151. doi: 10.1007/s00221-005-0076-0

Freitas, C., Fregni, F., and Pascual-Leone, A. (2009). Meta-analysis of the effects of repetitive transcranial magnetic stimulation (rTMS) on negative and positive symptoms in schizophrenia. Schizophr. Res. 108, 11-24. doi: 10.1016/j.schres.2008.11.027

Frye, R. E., Rotenberg, A., Ousley, M., and Pascual-Leone, A. (2008). Transcranial magnetic stimulation in child neurology: current and future directions. J. Child Neurol. 23, 79-96. doi: 10.1177/0883073807307972

Fulton, E., Eapen, V., Crnčec, R., Walter, A., and Rogers, S. (2014). Reducing maladaptive behaviors in preschool-aged children with autism spectrum disorder using the Early Start Denver Model. Front. Pediatr. 2:40. doi: 10.3389/fped.2014.00040

Gillick, B. T., Feyma, T., Menk, J., Usset, M., Vaith, A., Wood, T. J., et al. (2015). Safety and feasibility of transcranial direct current stimulation in pediatric hemiparesis: randomized controlled preliminary study. Phys. Ther. 95, 337-349. doi: 10.2522/ptj.20130565

Hasan, A., Bergener, T., Nitsche, M. A., Strube, W., Bunse, T., Falkai, P., et al. (2013). Impairments of motor-cortex responses to unilateral and bilateral direct current stimulation in schizophrenia. Front. Psychiatry 4:121. doi: 10.3389/fpsyt.2013.00121

Iyer, M. B., Mattu, U., Grafman, J., Lomarev, M., Sato, S., and Wassermann, E. M. (2005). Safety and cognitive effect of frontal DC brain polarization in healthy individuals. Neurology 64, 872-875. doi: 10.1212/01.WNL.0000152986.07469.E9

Jawaid, A., Riby, D. M., Owens, J., White, S. W., Tarar, T., and Schulz, P. E. (2012). 'Too withdrawn' or 'too friendly': considering social vulnerability in two neuro-developmental disorders. J. Intellect. Disabil. Res. 56, 335-350. doi: 10.1111/j.1365-2788.2011.01452.x
Jawaid, A., Schmolck, H., and Schulz, P. E. (2008). Hypersociability in Williams syndrome: a role for the amygdala? Cogn. Neuropsychiatry 13, 338-342. doi: 10.1080/13546800802243334

Jones, W., Bellugi, U., Lai, Z., Chiles, M., Reilly, J., Lincoln, A., et al. (2000). Hypersociability in Williams Syndrome. J. Cogn. Neurosci. 12, 30-46. doi: $10.1162 / 089892900561968$

Karmiloff-Smith, A., D’Souza, D., Dekker, T. M., Van Herwegen, J., Xu, F., Rodic, M., et al. (2012). Genetic and environmental vulnerabilities in children with neurodevelopmental disorders. Proc. Natl. Acad. Sci. U.S.A. 109(Suppl. 2), 17261-17265. doi: 10.1073/pnas.1121087109

Klein-Tasman, B. P., and Albano, A. M. (2007). Intensive, short-term cognitivebehavioral treatment of OCD-like behavior with a young adult with Williams syndrome. Clin. Case Stud. 6, 483-492. doi: 10.1177/1534650106296370

Korenberg, J. R., Chen, X. N., Hirota, H., Lai, Z., Bellugi, U., Burian, D., et al. (2000). VI. Genome structure and cognitive map of Williams syndrome. J. Cogn. Neurosci. 12(Suppl. 1), 89-107. doi: 10.1162/089892900562002

Laing, E., Butterworth, G., Ansari, D., Gsödl, M., Longhi, E., Panagiotaki, G., et al. (2002). Atypical development of language and social communication in toddlers with Williams syndrome. Dev. Sci. 5, 233-246. doi: 10.1111/14677687.00225

Lefaucheur, J. P., André-Obadia, N., Antal, A., Ayache, S. S., Baeken, C., Benninger, D. H., et al. (2014). Evidence-based guidelines on the therapeutic use of repetitive transcranial magnetic stimulation (rTMS). Clin. Neurophysiol. 125, 2150-2206. doi: 10.1016/j.clinph.2014.05.021

Magiati, I., Tay, X. W., and Howlin, P. (2014). Cognitive, language, social and behavioural outcomes in adults with autism spectrum disorders: a systematic review of longitudinal follow-up studies in adulthood. Clin. Psychol. Rev. 34, 73-86. doi: 10.1016/j.cpr.2013.11.002

Martens, M. A., Hasinski, A. E., Andridge, R. R., and Cunningham, W. A. (2012). Continuous cognitive dynamics of the evaluation of trustworthiness in Williams syndrome. Front. Psychol. 3:160. doi: 10.3389/fpsyg.2012.00160

Martens, M. A., Wilson, S. J., Dud- geon, P., and Reutens, D. C. (2009). Approachability and the amygdala: insights from Williams syndrome. Neuropsychologia 47, 2446-2453. doi: 10.1016/j.neuropsychologia.2009.04.017

McGurk, S. R., Twamley, E. W., Sitzer, D. I., McHugo, G. J., and Mueser, K. T. (2007). A meta-analysis of cognitive remediation in Schizophrenia. Am. J. Psychiatry 164, 1791-1802. doi: 10.1176/appi.ajp.2007.07060906

Mehta, U. M., Thirthalli, J., Basavaraju, R., and Gangadhar, B. N. (2014). Association of intracortical inhibition with social cognition deficits in schizophrenia: findings from a transcranial magnetic stimulation study. Schizophr. Res. 158, 146-150. doi: 10.1016/j.schres.2014.06.043

Mehta, U. M., Thirthalli, J., Subbakrishna, D. K., Gangadhar, B. N., Eack, S. M., and Keshavan, M. S. (2013). Social and neuro-cognition as distinct cognitive factors in schizophrenia: a systematic review. Schizophr. Res. 148, 3-11. doi: 10.1016/j.schres.2013.05.009

Mervis, C. B., Klein-Tasman, B. P., and Mastin, M. E. (2001). Adaptive behavior of 4-through 8-year-old children with Williams syndrome. Am. J. Mental Retard. 106, 82-96. doi: 10.1352/0895-8017(2001)106<0082:ABOTYO>2.0.CO;2

Meyer-Lindenberg, A., Hariri, A. R., Munoz, K. E., Mervis, C. B., Mattay, V. S., Morris, C. A., et al. (2005). Neural correlates of genetically abnormal social cognition in Williams syndrome. Nat. Neurosci. 8, 991-993. doi: $10.1038 / \mathrm{nn} 1494$

Mimura, M., Hoeft, F., Kato, M., Kobayashi, N., Sheau, K., Piggot, J., et al. (2010). A preliminary study of orbitofrontal activation and hypersociability in Williams Syndrome. J. Neurodev. Disord. 2, 93-98. doi: 10.1007/s11689-009-9041-8

Minhas, P., Bikson, M., Woods, A. J., Rosen, A. R., and Kessler, S. K. (2012). "Transcranial direct current stimulation in pediatric brain: a computational modeling study," in Engineering in Medicine and Biology Society (EMBC), 2012 Annual International Conference of the IEEE (San Diego, CA). doi: 10.1109/embc.2012.6346067

Minio-Paluello, I., Baron-Cohen, S., Avenanti, A., Walsh, V., and Aglioti, S. M. (2009). Absence of embodied empathy during pain observation in Asperger syndrome. Biol. Psychiatry 65, 55-62. doi: 10.1016/j.biopsych.2008.08.006

Miniussi, C., and Rossini, P. M. (2011). Transcranial magnetic stimulation in cognitive rehabilitation. Neuropsychol. Rehabil. 21, 579-601. doi: 10.1080/09602011.2011.562689

Muñoz, K. E., Meyer-Lindenberg, A., Hariri, A. R., Mervis, C. B., Mattay, V. S., Morris, C. A., et al. (2010). Abnormalities in neural processing of 
emotional stimuli in Williams syndrome vary according to social vs. nonsocial content. NeuroImage 50, 340-346. doi: 10.1016/j.neuroimage.2009. 11.069

Nitsche, M. A., Cohen, L. G., Wassermann, E. M., Priori, A., Lang, N., Antal, A., et al. (2008). Transcranial direct current stimulation: state of the art 2008. Brain Stimul. 1, 206-223. doi: 10.1016/j.brs.2008.06.004

Nitsche, M. A., Niehaus, L., Hoffmann, K. T., Hengst, S., Liebetanz, D., Paulus, W., et al. (2004). MRI study of human brain exposed to weak direct current stimulation of the frontal cortex. Clin. Neurophysiol. 115, 2419-2423. doi: 10.1016/j.clinph.2004.05.001

Palm, U., Keeser, D., Blautzik, J., Pogarell, O., Ertl-Wagner, B., Kupka, M. J., et al. (2013). Prefrontal transcranial direct current stimulation (tDCS) changes negative symptoms and functional connectivity MRI (fcMRI) in a single case of treatment-resistant schizophrenia. Schizophr. Res. 150, 583-585. doi: 10.1016/j.schres.2013.08.043

Pascual-Leone, A., Walsh, V., and Rothwell, J. (2000). Transcranial magnetic stimulation in cognitive neuroscience-virtual lesion, chronometry, and functional connectivity. Curr. Opin. Neurobiol. 10, 232-237. doi: 10.1016/S0959-4388(00)00081-7

Phillips, K. D., and Klein-Tasman, B. P. (2009). Mental health concerns in Williams syndrome: intervention considerations and illustrations from case examples. J. Ment. Health Res. Intellect. Disabil. 2, 110-133. doi: $10.1080 / 19315860802627601$

Pinkham, A. E., Penn, D. L., Perkins, D. O., and Lieberman, J. (2003). Implications for the neural basis of social cognition for the study of schizophrenia. Am. J. Psychiatry 160, 815-824. doi: 10.1176/appi.ajp.160.5.815

Preti, A., Melis, M., Siddi, S., Vellante, M., Doneddu, G., and Fadda, R. (2014). Oxytocin and autism: a systematic review of randomized controlled trials. J. Child Adolesc. Psychopharmacol. 24, 54-68. doi: 10.1089/cap. 2013.0040

Puzzo, I., Coopera, N. R., Vetterb, P., Russo, R., and Fitzgerald, P. B. (2009). Reduced cortico-motor facilitation in a normal sample with high traits of autism. Neurosci. Lett. 467, 173-177. doi: 10.1016/j.neulet.2009. 10.033

Riby, D., and Hancock, P. J. B. (2009). Looking at movies and cartoons: eyetracking evidence from Williams syndrome and autism. J. Intellect. Disabil. Res. 53, 169-181. doi: 10.1111/j.1365-2788.2008.01142.x

Riby, D. M., and Hancock, P. J. B. (2008). Viewing it differently: social scene perception in Williams syndrome and autism. Neuropsychologia 46, 2855-2860. doi: 10.1016/j.neuropsychologia.2008.05.003

Riby, D. M., Hanley, M., Kirk, H., Clark, F., Little, K., Fleck, R., et al. (2014). The interplay between anxiety and social functioning in Williams syndrome. J. Autism Dev. Disord. 44, 1220-1229. doi: 10.1007/s10803-013-1984-7

Rolf, L. H., Haarmann, F. Y., Grotemeyer, K. H., and Kehrer, H. (1993). Serotonin and amino acid content in platelets of autistic children. Acta Psychiatr. Scand. 87, 312-316. doi: 10.1111/j.1600-0447.1993.tb03378.x

Rosner, B. A., Hodapp, R. M., Fidler, D. J., Sagun, J. N., and Dykens, E. M. (2004). Social competence in persons with Prader-Willi, Williams and Down's syndromes. J. Appl. Res. Intellect. Disabil. 17, 209-217. doi: 10.1111/j.14683148.2004.00200.x

Rossi, S., Hallett, M., Rossini, P. M., Pascual-Leone, A., and Safety of TMS Consensus Group. (2009). Safety, ethical considerations, and application guidelines for the use of transcranial magnetic stimulation in clinical practice and research. Clin. Neurophysiol. 120, 2008-2039. doi: 10.1016/j.clinph.2009.08.016

Santiesteban, I., Banissy, M. J., Catmur, C., and Bird, G. (2012). Enhancing social ability by stimulating right temporoparietal junction. Curr. Biol. 22, 2274-2277. doi: 10.1016/j.cub.2012.10.018

Shi, C., Yu, X., Cheung, E. F., Shum, D. H., and Chan, R. C. (2014). Revisiting the therapeutic effect of rTMS on negative symptoms in schizophrenia: a meta-analysis. Psychiatry Res. 215, 505-513. doi: 10.1016/j.psychres.2013. 12.019

Sokhadze, E., Baruth, J., Tasman, A., Mansoor, M., Ramaswamy, R., Sears, L., et al. (2010). Low-frequency repetitive transcranial magnetic stimulation (rTMS) affects event-related potential measures of novelty processing in autism. Appl. Psychophysiol. Biofeedback 35, 147-161. doi: 10.1007/s10484-009-9121-2

Sokhadze, E. M., Baruth, J. M., Sears, L., Sokhadze, G. E., El-Baz, A. S., and Casanova, M. F. (2012). Prefrontal neuromodulation using rTMS improves error monitoring and correction function in autism. Appl. Psychophysiol. Biofeedback 37, 91-102. doi: 10.1007/s10484-012-9182-5

Sokhadze, E. M., El-Baz, A., Baruth, J., Mathai, G., Sears, L., and Casanova, M. F. (2009). Effects of low frequency repetitive transcranial magnetic stimulation (rTMS) on gamma frequency oscillations and event-related potentials during processing of illusory figures in autism. J. Autism Dev. Disord. 39, 619-634. doi: 10.1007/s10803-008-0662-7

Sokhadze, E. M., El-Baz, A. S., Sears, L. L., Opris, I., and Casanova, M. F. (2014). rTMS neuromodulation improves electrocortical functional measures of information processing and behavioral responses in autism. Front. Syst. Neurosci. 8:134. doi: 10.3389/fnsys.2014.00134

Stagg, C. J., and Nitsche, M. A. (2011). Physiological basis of transcranial direct current stimulation. Neuroscientist 17, 37-53. doi: 10.1177/1073858410386614

Théoret, H., Halligan, E., Kobayashi, M., Fregni, F., Tager-Flusberg, H., and Pascual-Leone, A. (2005). Impaired motor facilitation during action observation in individuals with autism spectrum disorder. Curr. Biol. 15, 84-85. doi: 10.1016/j.cub.2005.01.022

Tobin, M. C., Drager, K. D., and Richardson, L. F. (2014). A systematic review of social participation for adults with autism spectrum disorders: support, social functioning, and quality of life. Res. Autism Spectr. Disord. 8, 214-229. doi: 10.1016/j.rasd.2013.12.002

Warren, Z., Veenstra-VanderWeele, J., Stone, W., Bruzek, J. L., Nahmias, A. S., Foss-Feig, J. H., et al. (2011). Therapies for Children with Autism Spectrum Disorders. Comparative Effectiveness Review No. 26 (Prepared by the Vanderbilt Evidence-Based Practice Center under Contract No. 290-2007-10065-I). Rockville, MD: Agency for Healthcare Research and Quality. AHRQ Publication No. 11-EHC029-EF. Available online at: www. effectivehealthcare.ahrq.gov/reports/final.cfm (Accessed April, 2011).

Williams, J. H. G., Whiten, A., Suddendorf, T., and Perrett, D. I. (2001). Imitation, mirror neurons and autism. Neurosci. Biobehav. Rev. 25, 287-295. doi: 10.1016/S0149-7634(01)00014-8

Won, H., Mah, W., and Kim, E. (2013). Autism spectrum disorder causes, mechanisms, and treatments: focus on neuronal synapses. Front. Mol. Neurosci. 6:19. doi: 10.3389/fnmol.2013.00019

Young, L., Camprodon, J. A., Hauser, M., Pascual-Leone, A., and Saxe, R. (2010). Disruption of the right temporoparietal junction with transcranial magnetic stimulation reduces the role of beliefs in moral judgments. Proc. Natl. Acad. Sci. U.S.A. 107, 6753-6758. doi: 10.1073/pnas.0914826107

Zaghi, S., Heine, N., and Fregni, F. (2009). Brain stimulation for the treatment of pain: a review of costs, clinical effects, and mechanisms of treatment for three different central neuromodulatory approaches. J. Pain Manag. 2, 339-352.

Conflict of Interest Statement: The authors declare that the research was conducted in the absence of any commercial or financial relationships that could be construed as a potential conflict of interest.

Copyright (c) 2015 Boggio, Asthana, Costa, Valasek and Osório. This is an open-access article distributed under the terms of the Creative Commons Attribution License (CC $B Y)$. The use, distribution or reproduction in other forums is permitted, provided the original author(s) or licensor are credited and that the original publication in this journal is cited, in accordance with accepted academic practice. No use, distribution or reproduction is permitted which does not comply with these terms. 